\title{
Rationalisation of building maintenance through Markov decision models
}

\author{
Chris van Winden and Rommert Dekker \\ Econometric Institute, Erasmus University Rotterdam
}

In this paper we present Markov decision models for rationalisation of building maintenance. The models were developed for the Dutch State Building Agency, which is responsible for about 4000 state owned buildings with a value of some 20 billion guilders and an annual maintenance budget of some 90 million guilders. The models are intended to increase insight into strategic maintenance decision making and are in a preliminary phase only. Each building is divided into several parts, which state is determined each year by inspection and the result classified on a 6 point scale (varying from excellent condition to a very bad condition). Assuming residence times for each state, a semi-Markov decision model (SMDM) can be formulated with several maintenance actions in each state. From norms on the maximal discounted frequencies of certain states a minimum discounted cost policy can be determined by formulating the SMDM as a linear programming model. To incorporate the initial state of buildings and yearly budget constraints, however, the SMDM has to be transformed into an equivalent Markov decision model (MDM) with the residence time as second state variable. Modelling deterioration dependencies between e.g. protective parts (painting) and protected parts, however, leads to very large states spaces. Furthermore, a discounted cost analysis does not allow the control over the long term average state frequencies. Finally, by averaging over all building parts the MDM also allows the construction of a so-called tradeoff curve between the average condition of buildings and the long term average costs required to keep the buildings in that condition. 